\title{
Effect of isosorbide mononitrate on the human optic nerve and choroidal circulations
}

\author{
Juan E Grunwald, Alessandro Iannaccone, Joan DuPont
}

\begin{abstract}
Aims-To assess the effects of the nitric oxide donor 5-isosorbide mononitrate (ISMO) on blood flow in the optic nerve head (ON flow) and choroid (Ch flow).

Methods-Laser Doppler flowmetry was used to measure $\mathrm{ON}$ flow and $\mathrm{Ch}$ flow in 12 normal subjects by aiming the laser beam at the fovea and at the temporal rim, respectively. In a double masked, randomised, crossover design, each subject received orally on separate days either 20 mg of 5-isosorbide mononitrate (ISMO) or placebo. Ch flow and $\mathrm{ON}$ flow were determined monocularly at baseline and 1 hour after dosing. In the last six subjects, additional measurements were obtained at 3 hours. Mean arterial blood pressure (BPm), heart rate, and intraocular pressure (IOP) were monitored, and ocular perfusion pressure (PP) was estimated.

Results-No significant changes in ON flow, PP, IOP, or BPm were observed following placebo. Following ISMO, ON flow increased from baseline by $19.8 \%$ (SEM 9.3\%) at 1 hour (paired $t$ test, $\mathrm{p}=$ $0.058)$ and by $33.1 \%(7.5 \%)$ at 3 hours $(p=$ 0.007). Compared with the changes following placebo, statistically significant increases in $\mathrm{ON}$ flow were observed both at $1(p=0.050)$ and 3 hours $(p=0.041)$ after ISMO treatment. Compared with placebo, PP decreased significantly 1 hour after ISMO dosing $(p=0.039)$, mainly as a function of reduced $\mathrm{BPm}$. A significant inverse correlation $(R=-0.618 ; p=0.032)$ was $o b-$ served between the percentage changes in PP and ON flow 1 hour following ISMO administration, but not after placebo. No significant change in foveal $\mathrm{Ch}$ flow was documented.
\end{abstract}

Conclusions-These results suggest that, in normal subjects, ISMO increases significantly $\mathrm{ON}$ flow but not $\mathrm{Ch}$ flow. The inverse correlation observed between PP and $O N$ flow suggests that the same mechanism(s) responsible for systemic vasodilatation and blood pressure decrease may also cause the $O N$ flow increase.

(Br F Ophthalmol 1999;83:162-167)

Recent reports by Zurakowski et $a l^{1}$ and Afshari et $a l^{2}$ have suggested that glaucoma patients who receive nitrate therapy for systemic conditions unrelated to glaucoma have less progression of glaucomatous optic neuropathy and visual field function loss than patients that do not receive these compounds.
In an attempt to elucidate the possible mechanism(s) by which nitrates may protect from glaucomatous damage, Grunwald et $a l^{3}$ investigated whether chronic nitrate treatment was associated with retinal vasodilatation. Their study has shown that glaucoma patients receiving chronic nitrate therapy for cardiac conditions have significantly larger retinal vein diameters than glaucoma patients who were not receiving nitrate therapy. Possibly, vasodilatation could be associated with improved perfusion of the retina and perhaps the optic nerve, in a similar fashion to what has been observed in the circulation of the heart, providing a potential rationale for the observations reported by Zurakowski et $a l^{1}$ and Afshari et $a l^{2}$

Nitrates are well known vasodilating agents effective in a dose dependent manner on both veins and, to a lesser extent, arteries. ${ }^{4}$ Nitrates produce vasodilatation by acting as nitric oxide donors. ${ }^{4}$ The prototype of nitrates, nitroglycerin, has a rapid onset of action, but is rapidly cleared from the blood stream. ${ }^{4}$ Long acting nitrates such as isosorbide dinitrate (ISDN) are more suitable for chronic therapies, but first pass hepatic metabolism can limit bioavailability, requiring multiple dose administrations that stimulate tolerance. ${ }^{4-8}$

In the past few years, the long acting compound 5-isosorbide mononitrate (ISMO, Wyeth-Ayerst Laboratories, Philadelphia, PA, USA) has become available for use in clinical practice. ISMO is the active metabolite of ISDN, requiring no first pass hepatic metabolism, which guarantees nearly $100 \%$ bioavailability. ${ }^{7}$ Maximum serum concentrations are achieved in 45-60 minutes, peak effect is maintained between 1 and 4 hours, and half life is $4-5$ hours. ${ }^{4-7}$ The recommended regimen to attain therapeutic levels is $20 \mathrm{mg}$ twice daily. The high bioavailability limits the number of dose administrations required to attain therapeutic levels, and circumvents the risk for tolerance during long term nitrate treatment protocols. ${ }^{4-68}$

The effects of ISMO on the ocular microcirculation are unknown. We investigated the effects of a single dose of ISMO on the circulation of the choroid and optic disc vessels, in an attempt to assess the effect of nitrates on the human ocular circulation and their potential role in the management of ocular vascular diseases.

Materials and methods

Twelve healthy volunteers aged 19-37 years (mean 29 (SEM 1.7) years) were included in this study. All subjects had no history of 
Table 1 Pressure data (mean (SEM))

\begin{tabular}{|c|c|c|c|}
\hline Percentage change from baseline & Placebo & ISMO & $\begin{array}{l}\text { p Value } \\
\text { placebo v ISMO }\end{array}$ \\
\hline \multicolumn{4}{|l|}{ Mean blood pressure (BPm): } \\
\hline 1 hour $(\mathrm{n}=12)$ & $+0.3(2.8)$ & $-8.8(1.7)$ & 0.030 \\
\hline 3 hours $(n=6)$ & $+1.6(4.6)$ & $-5.9(5.2)$ & 0.284 \\
\hline \multicolumn{4}{|l|}{ Intraocular pressure (IOP): } \\
\hline 1 hour $(n=12)$ & $+1.2(2.8)$ & $+4.3(4.2)$ & 0.433 \\
\hline 3 hours $(n=6)$ & $+0.8(4.0)$ & $-6.1(7.0)$ & 0.431 \\
\hline \multicolumn{4}{|l|}{ Perfusion pressure (PP): } \\
\hline 1 hour $(n=12)$ & $+0.8(4.1)$ & $-11.9(2.9)$ & 0.039 \\
\hline 3 hours $(n=6)$ & $+3.6(6.1)$ & $-3.7(8.5)$ & 0.496 \\
\hline
\end{tabular}

*Paired, two tailed Student's $t$ test for the comparison between percentage changes following placebo and ISMO.

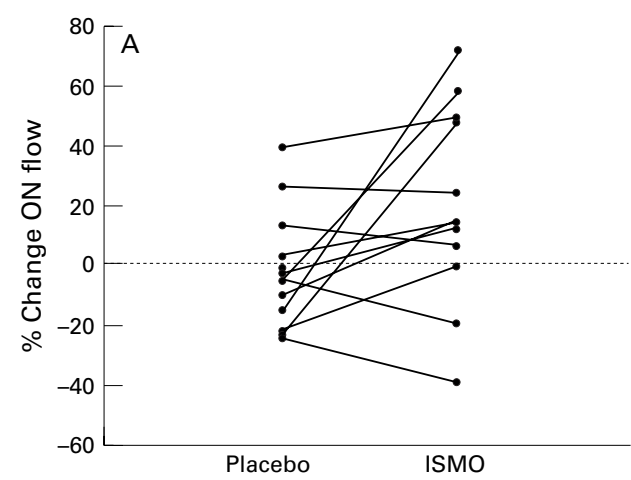

systemic or intraocular disease and were not taking any systemic or topical medications. Corrected visual acuities were $20 / 20$ and intraocular pressures (IOP) were $20 \mathrm{~mm} \mathrm{Hg}$. Slit lamp and dilated funduscopic examinations were normal in all subjects.

On the days when the experiments where carried out, participants were asked to abstain from smoking and drinking caffeine containing drinks until the end of the measurements. Informed consent was obtained from each subject after the procedures were explained. The protocol of this study had been approved by the institutional review board of our medical centre.

Pupils were dilated with tropicamide $1 \%$ (Alcon, Humacao, Puerto Rico), and Polaroid (Cambridge, MA, USA) colour fundus photographs of the discs were taken for accurate documentation of the location of the blood flow measurements. Laser Doppler flowmetry (LDF) was used to assess relative blood velocity, volume and flow in the optic nerve head and choroid. All measurements were performed in one eye (the study eye), which was determined at random.

LDF measurements were obtained with the Oculix LDF instrument (Philadelphia, PA, USA) based on a modified Topcon TRCJE fundus camera (Tokyo, Japan) which allows the delivery of a laser beam to any point of the posterior pole. A diode laser $(670 \mathrm{~nm})$ was used. Analysis of the data was performed using a NeXT computer (Redwood City, CA, USA) with software specifically developed for the analysis of Doppler signals from ocular tissues. Detailed descriptions of the technique have been previously published..$^{9-11}$ In brief, relative choroidal blood velocity ( $\mathrm{Ch}$ vel), volume ( $\mathrm{Ch}$ vol) and flow (Ch flow) were assessed by aiming the probing laser beam at the fovea, as previously described. ${ }^{9}$ Subsequently, LDF measurements of optic nerve head relative blood velocity, volume, and flow were obtained from the superior temporal and the inferior temporal neuroretinal rim in areas devoid of large vessels. Determinations at these two locations were averaged to provide a mean optic nerve head velocity (ON vel), volume ( $\mathrm{ON}$ vol) and flow (ON flow). ${ }^{10}{ }^{11}$ Measurements obtained with this technique have been estimated to reflect contributions not only from the superficial and prelaminar regions of the optic nerve head, but also from part of the laminar vessels. ${ }^{10}$

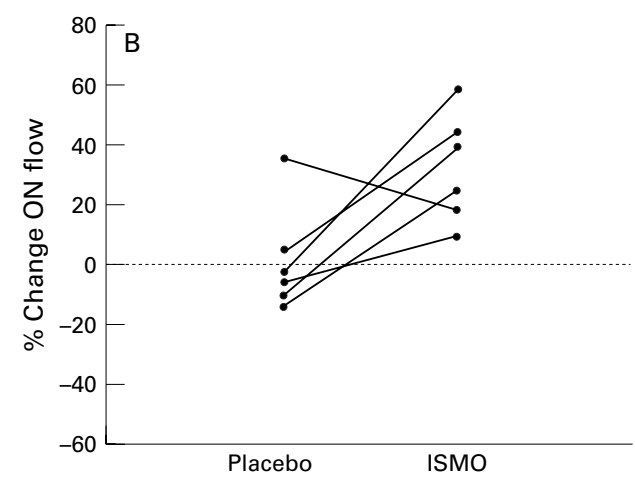

Figure 1 Comparison of the percentage changes from baseline in optic nerve head blood flow (ON flow) after placebo and ISMO treatment in each subject. (A) Measurements at 1 hour after dosing $(n=12)$. (B) Measurements at 3 hours after dosing $(n=6)$.

Immediately after LDF determinations, the systolic and diastolic blood pressures were measured by sphygmomanometry and the heart rate was determined. After instillation of two drops of topical proparacaine (proxymetacaine) $\mathrm{HCl} 0.5 \%$ (Allergan Pharmaceuticals, Hormigueros, Puerto Rico), IOP was measured in both eyes by Goldmann applanation tonometry. Mean brachial artery blood pressure (BPm) was calculated according to the formula $\mathrm{BPm}=2 / 3 \mathrm{BPd}+1 / 3 \mathrm{BPs}$, where $\mathrm{BPd}$ and BPs are the brachial artery diastolic and systolic pressures, respectively. Perfusion pressure $(\mathrm{PP})$ was estimated as $\mathrm{PP}=2 / 3 \mathrm{BPm}$ - IOP.

In a double masked, randomised, crossover design, each subject received orally either 20 $\mathrm{mg}$ of ISMO or placebo. In all individuals, the above measurements were repeated at 1 hour, the time when peak serum concentrations are attained..$^{4-7}$ In the last six subjects, an additional measurement was obtained 3 hours after dosing. Determinations of $\mathrm{ON}$ vel, ON vol, ON flow, Ch vel, $\mathrm{Ch}$ vol, and $\mathrm{Ch}$ flow were performed by one trained examiner, masked with regard to treatment regimen. Results are expressed as mean percentage variations from baseline (SEM). Normal distribution of the data was assessed with the Wilk-Shapiro test. Statistical evaluation of the results was performed using two tailed, paired Student's $t$ test, linear regression, and correlation analysis. Probability values $<0.05$ were considered as statistically significant. 
Table 2 Optic nerve head data (mean (SEM))

\begin{tabular}{|c|c|c|c|}
\hline Percentage change from baseline & Placebo & ISMO & $\begin{array}{l}\text { p Value } \\
\text { placebo v ISMO }\end{array}$ \\
\hline \multicolumn{4}{|l|}{ Blood velocity (ON vel): } \\
\hline 1 hour $(n=12)$ & $-2.0(2.6)$ & $+0.3(3.2)$ & 0.611 \\
\hline 3 hours $(n=6)$ & $-3.8(4.4)$ & $+9.6(8.9)$ & 0.172 \\
\hline \multicolumn{4}{|l|}{ Blood volume (ON vol): } \\
\hline 1 hour $(n=12)$ & $-0.2(6.0)$ & $+21.1(11.5)$ & 0.074 \\
\hline 3 hours $(n=6)$ & $+5.8(8.3)$ & $+24.6(11.8)$ & 0.323 \\
\hline \multicolumn{4}{|l|}{ Blood flow (ON flow): } \\
\hline 1 hour $(n=12)$ & $-2.1(5.8)$ & $+19.8(9.3)$ & 0.050 \\
\hline 3 hours $(n=6)$ & $+1.6(7.4)$ & $+33.1(7.5)$ & 0.041 \\
\hline
\end{tabular}

*Paired, two tailed Student's $t$ test for the comparison between percentage changes following placebo and ISMO.

\section{Results}

Table 1 summarises the average percentage changes from baseline in BPm, IOP, and PP at 1 and 3 hours following the administration of placebo and ISMO, respectively. There were no significant differences in the average percentage changes in IOP, BPm, or PP after placebo administration. One hour after ISMO treatment, there was a reduction in PP of $-11.9 \%(2.9 \%)$. This change in PP was mainly function of a reduction in BPm of $-8.8 \%$ $(1.7 \%)$. In comparison with the changes after placebo, the average percentage decreases in PP and BPm were statistically significant (paired, two tailed Student's $t$ test: $\mathrm{p}=0.039$ and 0.030 , respectively; $n=12$ ). At 3 hours, no significant differences in average percentage changes in PP or BPm were observed $(\mathrm{p}=0.496$ and 0.284 , respectively; $n=6)$. In comparison with placebo, IOP did not change significantly at any time following ISMO dosing ( $p=0.433$ at 1 hour, and $p=0.431$ at 3 hours).

Figure 1 shows a comparison in each subject of the percentage changes in ON flow following placebo and ISMO. ISMO produced an average $\mathrm{ON}$ flow increase from baseline of $19.8 \%(9.3 \%)(\mathrm{p}=0.058 ; \mathrm{n}=12)$ at 1 hour, and of $33.1 \%(7.5 \%)(p=0.007 ; n=6)$ at 3 hours. In comparison with the effects following placebo, the increases in $\mathrm{ON}$ flow were statistically significant at 1 hour $(p=0.050)$ and at 3 hours $(p=0.041$; Table 2). As summarised in Table 2, the increases in ON flow were mainly due to changes in $\mathrm{ON}$ vol of $+21.1 \%(11.5 \%)$ at 1 hour, and $+24.6 \%(11.8 \%)$ at 3 hours, since ON vel increased by only $+0.3 \%(3.2 \%)$ at 1 hour, and $+9.6 \%(8.9 \%)$ at 3 hours.

A significant inverse correlation was observed 1 hour after ISMO dosing between the percentage changes in $\mathrm{PP}$ and $\mathrm{ON}$ flow $(R=-0.618 ; \mathrm{p}=0.032$; Fig $2 \mathrm{~A})$. Larger reductions in PP were associated with larger

Table 3 Choroidal data (mean (SEM))

\begin{tabular}{llll}
\hline Percentage change from baseline & Placebo & ISMO & $\begin{array}{l}\text { p Value } \\
\text { placebo v ISMO }\end{array}$ \\
\hline $\begin{array}{lll}\text { Blood velocity (Ch vel): } \\
\quad \text { hour (n=12) }\end{array}$ & $+1.0(6.1)$ & $-3.0(3.4)$ & 0.562 \\
$\quad$ 3 hours (n=6) & $+2.3(5.5)$ & $-2.5(2.0)$ & 0.382 \\
Blood volume (Ch vol): & & & \\
$\quad$ 1 hour (n=12) & $+4.3(12.4)$ & $-0.7(7.5)$ & 0.972 \\
$\quad$ hours (n=6) & $-8.3(7.7)$ & $-1.1(5.0)$ & 0.586 \\
Blood flow (Ch flow): & & $-3.9(6.7)$ & 0.406 \\
$\quad$ hour (n=12) & $+8.2(11.0)$ & $-3.6(4.5)$ & 0.828 \\
3 hours (n=6) & $-6.4(9.5)$ &
\end{tabular}

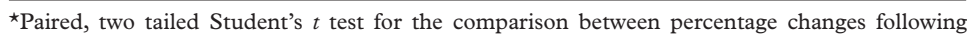
placebo and ISMO.
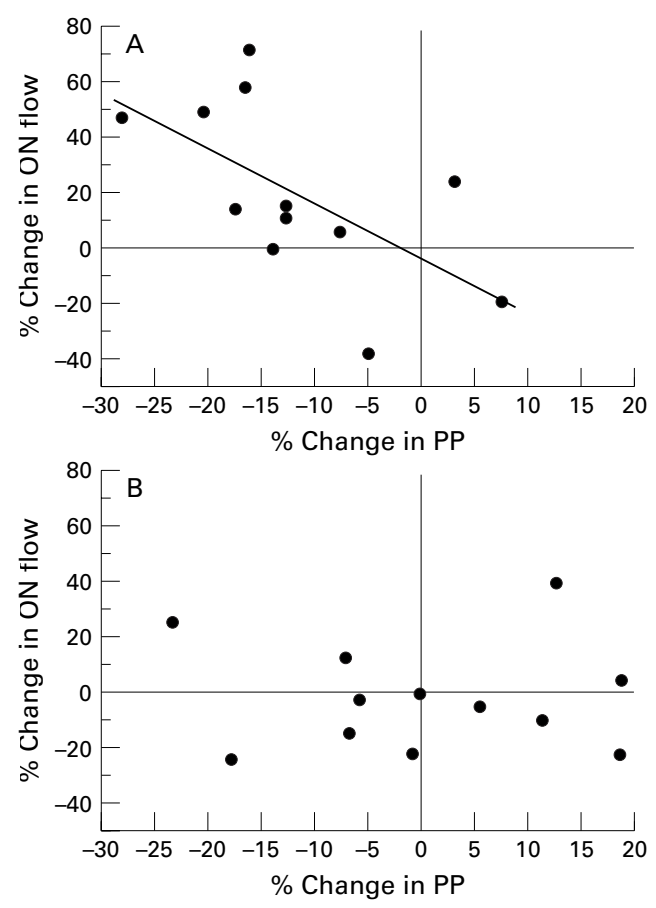

Figure 2 Percentage change in optic nerve head blood flow (ON flow) v percentage change in perfusion pressure $(P P)$ at 1 hour after ISMO $(A)$ and placebo $(B)$. There is a significant correlation after ISMO treatment $(R=-0.618$; $p=0.032)$, but not following placebo administration $(R=0.071 ; p=0.825)$.

increases in $\mathrm{ON}$ flow. In contrast, no significant correlation was observed 1 hour following placebo between the percentage changes in PP and ON flow ( $R=0.071 ; \mathrm{p}=0.825$; Fig $2 \mathrm{~B})$.

Table 3 summarises the average percentage changes from baseline in $\mathrm{Ch}$ vel, $\mathrm{Ch}$ vol, and Ch flow, at 1 and 3 hours after dosing with ISMO and placebo, respectively. In contrast with the changes observed for optic nerve data, no significant changes in any of the choroidal circulatory variables were observed following treatment.

After the administration of ISMO, seven subjects experienced side effects at 1 hour, and nine subjects at 3 hours. Mild to moderate headaches were reported by five subjects and sleepiness was noted by seven subjects (in three cases combined with headache). Two cases reported neck stiffness and one case asthenia. Headache peaked most often between 3 and 5 hours following ISMO dosing. Following placebo, no side effect was noted at 1 hour, whereas two subjects experienced mild diffuse headache at 3 hours.

We determined the reproducibility of our measurements by comparing baseline values obtained on the two different testing days. On the second day of measurement, baseline $\mathrm{Ch}$ flow varied by an average of $2.8 \%(8.2 \%)$ and ON flow by $0.9 \%(5.8 \%)$.

\section{Discussion}

Our findings suggest that ISMO causes in normal healthy volunteers an average increase in ON flow of nearly $20 \%$ at 1 hour and of $33 \%$ at 3 hours. In comparison with placebo, the average percentage increases in $\mathrm{ON}$ flow were 
statistically significant both at 1 hour $(p=0.050)$ and at 3 hours $(p=0.041)$ after ISMO administration.

Little is known about the effects of nitrates on the ocular circulation. Frayser and Hickam $^{12}$ studied the effects of sublingual nitroglycerin on the retinal circulation and reported dilatation of veins and arteries, reduced venous oxygen saturation, and increased mean circulation time. These results were interpreted as evidence suggesting a reduction in retinal blood flow. ${ }^{12}{ }^{13}$ On the other hand, Sinclair et al using colour Doppler ultrasound have documented nitroglycerin mediated increases in both systolic and diastolic retinal artery blood flow velocity. ${ }^{14}$ Experimental studies have shown the ability of nitroglycerin to recanalise retinal arterial occlusions, ${ }^{15}$ and anecdotal reports have suggested that sublingual nitroglycerin may improve blood flow in patients with central retinal artery occlusions. ${ }^{16}{ }^{17}$ To date, however, no study has evaluated the effects of nitrates on the optic nerve and choroidal circulations in human subjects.

Our findings following treatment with the long acting nitrate ISMO provide a potential rationale for the proposed beneficial effects of nitrergic drugs in glaucoma patients, ${ }^{12}$ but are in contrast with recent reports by Hessemer and Schmidt, ${ }^{18}{ }^{19}$ who observed a decrease in ocular pulse amplitude following administration of isosorbide dinitrate (ISDN), the parental molecule of ISMO. This decrease was interpreted as a probable reduction in ocular blood flow. ${ }^{18} 19$

There are several important differences between this study and ours that may account for the discrepancy in the results. Firstly, the techniques used to assess the flow changes are very different. Hessemer and Schmidt used an oculo-oscillo-dynamographic approach to estimate the ocular pulsation amplitude. ${ }^{18}{ }^{19}$ From this amplitude, they inferred information on the pulsatile component of total ocular blood flow, a step that is controversial. Their measurements could not provide information on the non-pulsatile ocular blood flow component, nor on the flow in specific vascular beds such as the optic nerve and the choroid.

Our laser Doppler flowmetry technique, ${ }^{9-11}$ on the other hand, is based on a completely different principle that allows a more direct and targeted measurement of flow changes in both the choroid and the optic nerve. This is an important difference, because we documented an effect of ISMO on ON flow but not on Ch flow. This suggests that an overall estimate of the ocular blood flow may not accurately reflect the effects that a vasoactive drug can exert on specific ocular vascular beds. A similar phenomenon has been previously noticed with $\mathrm{Ca}^{2+}$ channel blockers by Harino et al, ${ }^{20}$ who documented an effect of nicardipine on $\mathrm{ON}$ flow but not on retinal blood flow.

In addition, the ISDN formulation used by Hessemer and Schmidt is different from ISMO in two major aspects. ${ }^{18}{ }^{19}$ Firstly, ISDN was used as a sustained release drug, whereas we used ISMO in a prompt release formulation.
Most importantly, ISDN undergoes extensive first pass hepatic metabolism, which reduces bioavailability to only $20-25 \%{ }^{4}$ This makes ISDN active serum concentrations lower and less predictable, thereby affecting their impact on blood flow. By contrast, because of lack of significant first pass hepatic metabolism, ISMO guarantees a higher bioavailability and exerts its effect with a more consistent intensity and within a more predictable time window. ${ }^{4-8}$

Finally, although Hessemer and Schmidt concluded in their more recent report that caution may be warranted in using ISDN in glaucoma patients, ${ }^{19}$ they also acknowledged that a reduction in the pulsatile component of the ocular blood flow may also be produced by an increase in total ocular blood flow. Therefore, their finding of a reduced ocular pulse amplitude not only may not necessarily reflect a reduction in blood flow, but it may also be compatible with our finding of an increased flow at the optic nerve head.

As expected, ISMO produced a significant reduction in PP, mainly accounted for by a drop in BPm. A reduction in blood pressure has also been reported by Hessemer and Schmidt with ISDN, ${ }^{18}{ }^{19}$ and is consistent with the pharmacological effects of nitrates.

Of interest is the fact that we observed a significant inverse correlation between percentage changes in PP and ON flow at 1 hour $(n=12$, Fig 2A). In other words, subjects who showed larger reductions in PP tended to have larger increases in $\mathrm{ON}$ flow. These findings, which are the opposite of what one would expect in a passive vascular system, suggest that ISMO produced in the optic nerve head vasculature a local vasodilatory effect similar to that exerted on the systemic circulation. Most probably, due to this vasodilatation, $\mathrm{ON}$ flow increased in spite of the reduction in BPm and PP.

This hypothesis is supported by a large body of experimental evidence. Nitric oxide (NO), the vasodilating metabolite released by all nitrates, ${ }^{4}$ has a crucial role in the local regulation of ocular blood flow. ${ }^{21-26}$ Nitrates relax bovine ophthalmic arteries not only by inhibiting both $\mathrm{Ca}^{2+}$ release and influx, but also by decreasing the vascular contraction force. ${ }^{27}$ Increases in $\mathrm{ON}$ flow following systemic administration of endothelin-1 in cats have been attributed to a NO mediated local mechanism, independent of systemic haemodynamic effects. ${ }^{28}$ An important role of NO in the regulation of optic nerve head blood flow in response to flickering stimuli has been clearly demonstrated in cats and primates. ${ }^{29-31}$

Consistent with these experimental findings, NO synthase (NOS) immunoreactivity has been documented in the optic nerve head. ${ }^{32} 33$ In normal human eyes, the endothelial isoform (eNOS) was expressed primarily in prelaminar disc vessels, ${ }^{33}$ thereby implying possible regional differences in the NO dependent regulation of optic nerve head blood flow. In optic nerves of glaucomatous eyes, prelaminar eNOS upregulation and ectopic eNOS expression at the level of the lamina cribrosa was observed. ${ }^{33}$ Therefore, a putative direct effect of ISMO, and in general of all nitrates, on 
eNOS positive optic nerve head vessels may account for the effects observed in our study, as well as those reported by Zurakowski et $a l^{1}$ and Afshari et $a l^{2}$ on glaucomatous patients.

Although NO is known to have a neurotoxic potential, ${ }^{26}$ and there is concern that increased NOS immunoreactivity at the optic nerve head level may correlate with disease progression in glaucoma, ${ }^{33}$ the role of NO in ocular disease remains uncertain. ${ }^{26}{ }^{33}$ At the retinal level, it has been shown that NO dependent excitotoxicity is mediated exclusively by neuronal NOS, and not by eNOS. ${ }^{34}$ Our findings support the possibility that, besides a possible neurotoxic effect mediated by neuronal NOS activation, increased NO availability induced by systemic administration of nitrates may exert a favourable effect by producing local vasodilatation and increased optic nerve head blood flow.

The more pronounced effect of ISMO on ON flow at 3 hours is somewhat surprising, because maximum serum ISMO concentrations are expected within 1 hour, and peak effect should be maintained between 1 and 4 hours. ${ }^{4-7}$ It is possible that the maximum effect in the central nervous system may be delayed, thereby explaining the phase lag between the expected systemic peak and the observed local ocular response. This may also explain why side effects, and particularly headaches, were more pronounced between 3 and 5 hours after ISMO dosing.

In contrast with the significant increase in ON flow observed in our study following ISMO, we did not find any significant change in average $\mathrm{Ch}$ flow in the fovea. This was not entirely surprising, because there is experimental evidence that choroidal vessels may be physiologically exposed to constitutively high NO levels. ${ }^{356}$ Therefore, it is possible that additional NO availability provided by long acting nitrates may be unable to elicit further choroidal dilatation in normal subjects. A word of caution is warranted here regarding the interpretation of our choroidal measurements. Because these determinations were obtained in the centre of the foveola, they may not be representative of what occurs in the more peripheral choroid.

In response to the significant reduction in $\mathrm{BPm}$ and PP following ISMO dosing, a decrease in $\mathrm{Ch}$ flow would have been expected if the choroid behaved as a passive vascular system. The lack of a significant $\mathrm{Ch}$ flow reduction may be due to the fact that the small decrease in BPm and PP may produce small changes in $\mathrm{Ch}$ flow that may not be detectable by our technique, or that perhaps the drug may produce some choroidal vasodilatation that could counteract the decrease in BPm and PP. A third possibility is that the lack of Ch flow change may be attributed to a choroidal autoregulatory response, at least in the foveal region. This hypothesis is consistent with recent evidence obtained with our same technique by Riva et $a l,{ }^{37}$ who did not observe Ch flow changes in healthy subjects despite marked decreases in PP. The possibility of a neural control of the subfoveal choroidal blood flow suggested by Riva and co-workers' findings ${ }^{37}$ and ours is well supported by the histological evidence of a dense nitrergic neural plexus in the human subfoveal choroid, which has been postulated to have a role in the regulation of local blood flow in this region. ${ }^{38}$

In conclusion, our results suggest a significant ON flow increase after a single ISMO dosing in young healthy subjects, in line with experimental data indicating an important role of nitric oxide in the regulation of ON flow. ${ }^{28-31}$ This increase was mainly mediated by an increment in blood volume, a finding that is consistent with the vasodilating effect of nitrates. Further studies are needed to elucidate whether this acute effect of ISMO on ON flow can be replicated in older patients with glaucoma and ischaemic optic neuropathies, and whether this effect may be maintained following long term ISMO administration.

The authors thank Dr Maureen Maguire for her statistical consultation, which was supported through grant NEI R21 EY10964.

Supported by the Foundation Fighting Blindness, the Vivian S Lasko Vascular Research Fund, and NIH grants NEI EY11479 and R21 EY10964.

The authors do not have any proprietary interests in any products mentioned in this paper.

1 Zurakowski D, Vorwerk CK, Gorla M, et al. Nitrate therapy may retard glaucomatous optic neuropathy perhaps may retard glaucomatous optic neuropathy perhaps press).

2 Afshari NA, Zurakowski D, Dreyer EB. The effect of long Afshari NA, Zurakowski D, Dreyer EB. The effect of long
acting nitrate preparations on glaucomatous optic neuropathy. (ARVO abstracts) Invest Ophthalmol Vis Sci 1997; ropathy. (ARVO

3 Grunwald JE, DuPont J, Dreyer EB. Effect of chronic nitrate treatment on retinal vessel caliber in open-angle glaucoma. Am 7 Ophthalmol 1997;123:753-8.

4 Abrams J. Long-acting nitrates in cardiovascular disease. In: Giles TD, Garbus SB, eds. Use of longer-acting agents in cardiac therapy. Recent advances. USA: Health Care Communications, Inc, 1989;107-28.

5 Abshagen UWP. Pharmacokinetics of isosorbide mononitrate. Am f Cardiol 1992;70:61G-6G.

6 Hutt V, Theodor R, Pabst G, et al. Evaluation of bioavailability and pharmacokinetics of two isosorbide-5mononitrate preparations in healthy volunteers. $\mathcal{F}$ Clin Pharmacol 1992;32:553-7.

7 Schaumann N. Pharmacokinetics of isosorbide dinitrate and isosorbide-5-mononitrate. Int $\mathcal{F}$ Clin Pharmacol Ther Tox isosorbide-5-mon

8 Thadani U, Maranda CR, Amsterdam E, et al. Lack of pharmacological tolerance and rebound angina pectoris during wice-daily therapy with isosorbide-5-mononitrate. Ann Intern Med 1994;120:353-9.

9 Riva CE, Cranstoun SD, Grunwald JE, et al. Choroidal blood flow in the foveal region of the human ocular fundus. Invest Ophthalmol Vis Sci 1994;35:4273-81.

10 Riva CE, Harino S, Petrig BL, et al. Laser-Doppler flowmetry in the optic nerve. Exp Eye Res 1992;55:499506.

11 Petrig BL, Riva CE. Optic nerve head laser Doppler flowmetry: principles and computer analysis. In: Kaiser, HJ, Flammer J, Hendrickson P, eds. Ocular blood flow. Basle: Karger, 1996:120-7.

12 Frayser R, Hickam JB. Effect of vasodilator drugs on the retinal blood flow in man. Arch Ophthalmol 1965;73:640-2.

13 Hickam JB, Frayser R. Studies of the retinal circulation in man. Observations on vessel diameter, arteriovenous oxygen difference, and mean circulation time. Circulation 1966;33:302-16.

14 Sinclair A-M, Hughes AD, Sever PS. Effect of nifedipine and glyceryl trinitrate on retinal blood flow in normal subjects. F Hum Hypertens 1993;7:399-401.

15 Wizemann A, Maier T, Wizemann V. Wirksamkeit unterschiedlicher Therapieprinzipien bei experimentellen Netzhautgefä $\beta$-verschlüssen. Fortschr Ophthalmol 1983;80: $176-8$.

16 Kuritzky S. Nitroglycerin to treat acute loss of vision. [Letter] N Engl f Med 1990;323:1428.

17 Charness ME, Liu GT. Central retinal artery occlusion in giant cell arteritis: treatment with nitroglycerin. Neurology 1991;41:1698-9.

18 Hessemer V, Schmidt KG. Einfluss des Vasodilatators Isosorbiddinitrat auf den okularen Kreislauf. Klin Monatsbl Augenheilkd 1996;208:1880-195.

19 Hessemer V, Schmidt KG. Influence of the vasodilator drug isosorbide dinitrate on ocular circulation. Arch Ophthalmol 1997;115:324-7.

20 Harino S, Riva CE, Petrig BL. Intravenous nicardipine in cats increases optic nerve but not retinal blood flow. Invest Ophthalmol Vis Sci 1992;33:2885-90. 
21 Yao K, Tschudi M, Flammer J, et al. Endotheliumdependent regulation of vascular tone of the porcine 8.

22 Haefliger IO, Flammer J, Luscher TF. Nitric oxide and endothelin-1 are important regulators of human ophthalmic artery. Invest Ophthalmol Vis Sci 1992;33:2340-3.

23 Haefliger IO, Zschauer A, Anderson DR. Relaxation of retinal pericyte contractile tone through the nitric oxide-cyclic guanosine monophosphate pathway. Invest Ophthalmol Vis Sci 1994;35:991-7.

24 Meyer P, Flammer J, Luscher TF. Endothelium-dependent regulation of the ophthalmic microcirculation in the perfused porcine eye: role of nitric oxide and endothelins. Invest Ophthalmol Vis Sci 1993;34:3614-21.

25 Kitamura Y, Okamura T, Kani K, et al. Nitric oxidemediated retinal arteriolar and arterial dilatation induced by substance P. Invest Ophthalmol Vis Sci 1993;34:2859-65.

26 Goldstein IM, Ostwald P, Roth S. Nitric oxide: a review of its role in retinal function and disease. Vision Res 1996;36: 2979.

27 Hiroishi G, Kobayashi S, Nishimura J, et al. Differential effects of diltiazem and nitroglycerin on cytosolic $\mathrm{Ca}^{2+}$ concentration and on force in the bovine ophthalmic artery. centration and on force in the bovine ophth
Invest Ophthalmol Vis Sci 1996;37:2612-23.

28 Nishimura K, Riva CE, Harino S, et al. Effects of endothelin-1 on optic nerve head blood flow in cats. $f \mathrm{Ocul}$ Pharmacol Ther 1996;12:75-83.

29 Buerk DG, Riva CE, Cranstoun SD. Nitric oxide has a vasodilatory role in cat optic nerve head during flicker stimuli. Microvasc Res 1996;52:13-26.
30 Kondo M, Wang L, Bill A. Vascular reponses in retina and optic nerve to L-NAME, a nitric oxide blocker, and flicker-
ing light in monkeys. ARVO Abstracts. Invest Ophthalmol Vis Sci 1995; 36(Suppl):477.

31 Kondo M, Wang L, Bill A. The role of nitric oxide in hyperaemic response to flicker in the retina and optic nerve in cats. Acta Ophthalmol Scand 1997;75:232-5.

32 Qi X, Guy J. Localization of NADPH diaphorase/nitric oxide synthase in the optic nerve of the normal guinea pig: a light and electron microscopic study. $f$ Comp Neurol 1996;370:396-404.

33 Neufeld AH, Hernandez MR, Gonzalez M. Nitric oxide synthase in the human glaucomatous optic nerve head. Arch Ophthalmol 1997;115:497-503.

34 Vorwerk CK, Hyman BT, Miller JW, et al. The role of neuronal and endothelial nitric oxide synthase in retinal excitotoxicity. Invest Ophthalmol Vis Sci 1997;38:20382044 .

35 Mann RM, Riva CE, Stone RA, et al. Nitric oxide and choroidal blood flow regulation. Invest Ophthalmol Vis Sci 1995;36:925-30.

36 Zagvazdin YS, Fitzgerald MEC, Sancesario G, et al. Neural nitric oxide mediates Edinger-Westphal nucleus evoked increase in choroidal blood flow in the pigeon. Invest $O p h-$ thalmol Vis Sci 1996;37:666-72.

37 Riva CE, Titze P, Hero M, et al. Effect of acute decreases of perfusion pressure on choroidal blood flow in humans. Invest Ophthalmol Vis Sci 1997;38:1752-60.

38 Flügel C, Tamm ER, Mayer B, et al. Species differences in choroidal vasodilative innervation: evidence for specific intrinsic nitrergic and VIP-positive neurons in the human eye. Invest Ophthalmol Vis Sci 1994;35:592-9. 